\title{
The Role of Fourier Series in Mathematics and in Signal Theory
}

\author{
Frode Rønning ${ }^{1}$ (DD
}

Accepted: 28 January 2021/ Published online: 10 June 2021

(C) The Author(s) 2021

\begin{abstract}
This paper presents a praxeological analysis, based on ATD (Anthropological Theory of the Didactic), of the topic of Fourier series, as this topic is introduced and used in mathematics and in electrical engineering, more precisely in signal theory. The analysis is based mainly on one widely used textbook in mathematics for engineers, and a textbook and video lectures in signal theory. The aim of the analysis is to investigate possible differences in the motivation for introducing Fourier series in mathematics and in signal theory, as well as differences in the techniques used, and the justifications behind the techniques. This research is inspired by previous research showing lack of connection between mathematics courses and engineering courses applying mathematics. To get a wider perspective, the exposition in the textbook in mathematics for engineers is also compared to the exposition in more advanced books in mathematics. To add to the perspective, the historical origin of Fourier series is also discussed. The findings show that there are differences in the motivation for the topic both between mathematics and signal theory, but also between basic and more advanced mathematics. Within mathematics there are also differences in the underlying theories.
\end{abstract}

Keywords Fourier series · Mathematics for engineers · Signal theory · ATD · Praxeology

\section{Introduction}

Mathematics taught as a service subject to engineering students is in many universities a large enterprise, and engineering students are probably the largest group of students taking part in mathematics classes at universities worldwide. Even if it is widely recognised that engineering students should learn mathematics, there are several answers to the questions of what the content of this mathematics should be for

Frode Rønning

frode.ronning@ntnu.no

1 Department of Mathematical Sciences, Norwegian University of Science and Technology, Trondheim, Norway 
different groups of engineering students, how it should be taught, and who should teach it. In a recent report, Alpers (2020) gives a state-of-the-art account of the role of mathematics as a service subject at the tertiary level. Alpers shows that the question of what mathematical content should be taught to students in the physical and natural sciences goes back at least to a meeting organised by the International Commission on Mathematical Instruction (ICMI) in 1911 (Alpers, 2020, p. 5). Later, questions have become more diverse, to include questions about to what extent the mathematics could or should be directly related to engineering subjects, how it should be taught, and by whom (Bajpaj, 1985). It seems that still no unified answers to these questions have been found (Flegg, Mallet, \& Lupton, 2012). At many universities mathematics is taught in courses specially designed for particular engineering programmes, by teachers dedicated to teaching students at this particular engineering programme. This model will provide good opportunities for including programme specific problems in the mathematics teaching, and it is assumed that this will increase the perceived relevance of mathematics (Alpers, 2008; Enelund, Larsson, \& Malmqvist, 2011). At other universities mathematics is taught to engineering students as part of a package of general courses, often in the first two years, with engineering courses only appearing later (Winkelman, 2009). It is claimed that this often leads to mathematics being taught with a focus only on mathematical concepts and understanding and not on applications (Loch \& Lamborn, 2016).

In many countries, engineering programmes face high drop-out rates, and not coping with mathematics may be an important reason for students dropping out. Many engineering students see mathematics as irrelevant, as something that takes the focus away from the engineering subjects (Loch \& Lamborn, 2016). Also, many students experience lack of connection between mathematics and engineering topics in the beginning. When they later need mathematics in the engineering courses they find it challenging to apply the mathematics they are supposed to have learned (Carvalho \& Oliveira, 2018; Harris, Black, Hernandez-Martinez, Pepin, \& Williams, 2015). To learn more about the reasons why students experience lack of connection it is of interest to analyse mathematical topics taught to different student groups by asking questions like "why is this topic taught to this particular group of students?", or "what aspects of this topic are important for this particular group of students?" My focus in this paper is on questions of this type. I will compare the topic of Fourier series, as this is presented in mathematics with the presentation of the same topic in electrotechnics, more specifically in signal theory. I will also compare the presentation in basic mathematics to the presentation in more advanced mathematics.

I base my analysis on the Anthropological Theory of the Didactic, abbreviated ATD, (Bosch \& Gascón, 2014; Chevallard, 2006). In this theory, knowledge is seen as being relative to the context, the institution, in which it appears (Bosch \& Gascón, 2014). Seeing mathematics and signal theory as different institutions I will investigate how Fourier series are treated both in mathematics and in signal theory. In ATD terms this can be expressed as investigating the praxeologies in these two institutions (Bosch \& Gascón, 2014). The investigation is based on how the topic of Fourier series is presented in common international textbooks, both in mathematics and in signal theory. Also, other learning resources (video lectures) in signal theory will be analysed. For a better understanding of the situation, I will also take a brief look at the historical origin of Fourier series, as well as the role of Fourier series in more advanced mathematics. 
This will, as it turns out, lead to the view that it may be reasonable to talk about several institutions also within mathematics. Special emphasis will be put on analysing the motivation for teaching Fourier series, and the reasoning and justification behind important results, both inside mathematics and when mathematics is used in signal theory. My aim is to answer the following research question: What characterises the praxeologies connected to Fourier series in mathematics and in signal theory? Results from such investigations can be useful when designing mathematics courses in engineering education and may be valuable in order to bridge the gap between theory and applications (Carvalho \& Oliveira, 2018; Harris et al., 2015).

\section{Theory}

The Anthropological Theory of the Didactics (ATD) is a research programme where the institutional aspect of teaching and learning mathematics is central. A central notion in ATD is the notion of praxeology, "the basic unit into which one can analyse human action at large" (Chevallard, 2006, p. 23). A fundamental principle in ATD is that no praxis, no human action, can exist without being explained or justified in certain ways, i.e. without a logos. Chevallard expresses this principle by stating that " $[p]$ raxis thus entails $\log o s$, which in turn, backs up praxis" (2006, p. 23). The praxis block $(P)$ consists of two parts, types of tasks $(T)$ and a set of techniques $(\tau)$ to carry out the tasks. The logos block $(L)$ also consists of two parts, a technology $(\theta)$, or justification for the techniques used to carry out the tasks, and the theory $(\Theta)$, which provides the basis and support for the technological discourse (Bosch \& Gascón, 2014 , p. 68). For short, the two blocks are written $P=[T, \tau]$ and $L=[\theta, \Theta]$. For the whole praxeology I will use the notation $\mathscr{P}=[P / L]=[T, \tau, \theta, \Theta]$, often referred to as the 4 T-model. In my analyses, notation in terms of signs and symbols will turn out to differ somewhat in the praxeologies that I study. Signs and symbols are important tools to carry out tasks in mathematics, and for this reason I will consider sign and symbols as part of the techniques, $\tau$.

A social situation is called a didactic situation

whenever one of its actors $(Y)$ does something to help a person $(x)$ or a group of persons $(X)$ learn something (indicated by a heart $\bullet$ ). A didactic system $S(X ; Y ; \boldsymbol{\vee})$ is then formed. The thing that is to be learned is called a didactic stake $\boldsymbol{}$ and is made up of questions or praxeological components. (Bosch \& Gascón, 2014, p. 71)

In my case $X$ can be seen as made up of students at an engineering programme where signal theory is an important part. $Y$ is made up of two components, $Y_{M}$ and $Y_{E}$, where $Y_{M}$ consists of teachers and learning resources involved in the teaching and learning of mathematics to $X$, and $Y_{E}$ consists of the corresponding components in an engineering course including signal theory as a topic, taught to the same group of persons $X$. The thing to be learned is Fourier series, and this topic will be treated in the learning resources both for mathematics and for the engineering course. I view the learning resources, and the courses for which they are used, as situated in two different institutions, $I_{M}$ and $I_{E}$, with their corresponding didactic stakes $\boldsymbol{\nabla}_{M}$ and $\boldsymbol{\nabla}_{E}$. Since the topic of Fourier series is explicitly treated in $I_{E}$ and not just carried over from $I_{M}$, it is reasonable to expect that $\boldsymbol{v}_{E} \neq \boldsymbol{\varphi}_{M}$, even if the topic is the same. Hence, there are two didactic systems, $S\left(X ; Y_{M} ; \boldsymbol{\vee}_{M}\right)$ and $S\left(X ; Y_{E} ; \boldsymbol{\vee}_{E}\right)$, 
and therefore two praxeologies, one for mathematics, $\mathscr{P}_{M}=\left[T_{M}, \tau_{M}, \theta_{M}, \Theta_{M}\right]$, and another for signal theory, $\mathscr{P}_{E}=\left[T_{E}, \tau_{E}, \theta_{E}, \Theta_{E}\right]$.

According to Chevallard (2006), the purpose of praxeologies is that they are needed to solve problems, or to answer questions: "The basic situation in this respect can be summed up like this: a question $Q$ is raised, and an answer $A$ is searched for" (Chevallard, 2006, p. 27). The question is dependent on the institution $(I)$ it emerges within and an answer to the question will be sought using an adequate praxeology $\mathscr{P}=$ $[P / L]$. I will refer to the question $Q$ in a praxeology as the generating question. Since the purpose of a praxeology is to solve a problem, it is important to have in mind what problems in the praxeology a particular topic is expected to solve.

In this paper I will investigate the praxeologies connected to Fourier series in the two institutions $I_{M}$ and $I_{E}$ to get an impression of how different they are. I will start by discussing the first three Ts in the $4 \mathrm{~T}$-model, $T$, $\tau$, and $\theta$, for the two praxeologies, and later I will discuss the theory, $\Theta$. An a priori assumption might be that the tasks are different, $T_{E} \neq T_{M}$, since the learning goals in the two subjects are different, and an important purpose of tasks is to help students reach the learning goals. Regarding the other Ts it is not so obvious to make an a priori assumption. I will also look at the generating questions, $Q_{E}$ and $Q_{M}$, in the two praxeologies to see which problems Fourier series are intended to solve in signal theory and which problems they are intended to solve in mathematics. Possible differences here may influence the perceived relevance of the topic for students.

It turns out to be too simplistic to talk about one mathematical praxeology. I will therefore distinguish between mathematics as taught to engineers and mathematics at a more advanced level. I will refer to the institution connected to mathematics for engineers by $I_{B M}$ (basic mathematics) and the institution where Fourier series appear at a more advanced level by $I_{A M}$ (advanced mathematics). Corresponding to this, I will refer to two mathematical praxeologies, $\mathscr{P}_{B M}=\left[T_{B M}, \tau_{B M}, \theta_{B M}, \Theta_{B M}\right]$ and $\mathscr{P}_{A M}=\left[T_{A M}\right.$, $\tau_{A M}, \theta_{A M}, \Theta_{A M}$, with potentially two different generating questions, $Q_{B M}$ and $Q_{A M}$. It could be argued that even this is too simplistic, since there is also basic mathematics for non-engineering students. However, my interest here is in engineering students, and therefore I take $B M$ to mean basic mathematics for engineering students.

\section{Previous Relevant Research}

Gueudet and Quéré (2018) raise the question about how teaching of mathematics can answer to the needs of engineering courses, and what the features of such teaching should be. Flegg et al. (2012) mention that there are different views regarding the degree of rigour and formality in engineering mathematics. They argue that "[w]ithout the explicit connection between theory and practice, the mathematical content of engineering programs may not be seen by students as relevant" (Flegg et al., 2012, p. 718). They also claim that in cases where mathematics departments teach the mathematical content to the engineering students, the engineering departments may have little idea of what mathematical content the students are exposed to.

Biehler, Kortemeyer and Schaper (2015) investigated a first-year course in electrical engineering, analysing tasks that required knowledge and cognitive resources both from mathematics and from electrical engineering. They identified instances where 
there was a gap between the mathematics learned, at school or at the university, and the mathematics needed for solving engineering tasks. Gueudet and Quéré (2018) emphasise the need for making connections and discuss connectivity on several levels. Here, connectivity on the micro-level could be for example connections between different topic areas, between different semiotic representations, and between different concepts.

Recently several researchers have shown how ATD can be a useful tool for investigating mathematics for user groups, such as engineering students. Hochmuth, Biehler and Schreiber (2014) discuss and contrast the use of modelling cycles with the use of ATD for studying epistemic relations between mathematics in higher mathematics courses and mathematics in engineering courses. They argue that ATD might be a relevant tool to use for example to address questions like "[w] hich meanings are actualized in the context of specific tasks and situations?" (Hochmuth et al., p. 697). Peters, Hochmuth and Schreiber (2017) use what they refer to as the extended praxeological ATD-model to analyse the relationship between different mathematical discourses in engineering courses, such as Signals and System Theory. Here, the $4 \mathrm{~T}$-model is extended in the sense that the techniques and technologies are split in two branches, based on whether the justification comes from electrotechnical or physical reasoning, or from mathematical reasoning. This is similar to my distinction between the didactic systems and corresponding praxeologies $\mathscr{P}_{B M}$ and $\mathscr{P}_{E}$.

Of particular relevance for my study are several recent papers by González-Martín and Hernandes-Gomes (2017, 2018, 2019a, b). These authors have compared presentations in Calculus textbooks with presentations in textbooks for professional engineering courses to see to what extent the concepts and techniques from Calculus are required to cope with the tasks in the engineering topics. I will now give a brief account of their work.

In the first of the range of papers (González-Martín \& Hernandes-Gomes, 2017), the authors look at the use of integrals in connection with bending moments in civil and mechanical engineering. An important part of their analysis is based on analysing parts of a textbook in Calculus and parts of a textbook in a Strength of Materials course, used for the same students. They found that although the notion of integral was used to teach the topic of bending moments in the engineering course, the techniques mostly relied on geometrical considerations. They found that the textbook in the engineering course avoided using notation and properties institutionalised in Calculus and concluded that the way integrals are taught in the Calculus course follows mathematical praxeologies that are too far from the way integrals are used in professional courses. In two other papers, (González-Martín \& Hernandes-Gomes, 2018, 2019a), the same authors investigate the use of integrals for computing first moments for plane regions in a civil engineering course. Also in this case they found that the tasks and techniques developed in the engineering course did not arise from mathematics but from an engineering discourse, and that it is only in the technology $(\theta)$ that integrals appear (GonzálezMartín \& Hernandes-Gomes, 2019a, p. 283). In the most recent paper, (GonzálezMartín \& Hernandes-Gomes, 2019b), the authors have extended their investigations to the use of integrals in electromagnetism. It seems that the results also in this case point in the same direction as in the previous investigations. Integrals are used to define the notions in question, but the tasks can be solved using geometric considerations, tables and ready-to-use formulas (González-Martín \& Hernandes-Gomes, 2019b). Hence, it is the conceptual aspects of the integral that are important, not the calculation techniques.

There are also interesting studies not using ATD comparing textbooks in mathematics with textbooks used in engineering areas. As an example, Alpers (2017) 
investigated two textbooks in engineering statics, one from the US and one from Germany, and compared the presentation in these books to what he described as "the usual treatment in mathematics textbooks" (Alpers, 2017, p. 137). This means that he did not consult one specific textbook in mathematics but he relied on his perception of what would be the common treatment in mathematics books. Performing a document analysis of the two textbooks from engineering, he found essential differences with respect to the concept, construction and notation of vectors, as well as the usage of differentials and the concept and notation of virtual displacements. He claims that the differences between the presentation in the investigated textbooks and a standard presentation in mathematics contain potential for cognitive mismatches between students' learning in mathematics and in statics (Alpers, 2017, p. 140).

The work on mechanical engineering mentioned above indicates a rather large gap between praxeologies in mathematics and parts of engineering. I will add to the existing literature by comparing praxeologies in mathematics and signal theory.

\section{Methodology}

My main sources of data for the analysis are two widely used textbook, one in mathematics (Kreyzig, 2011), in its 10th edition, and one in electrotechnology (Nilsson \& Riedel, 2011), in its 9th edition. These two books have a wide international distribution, and they have both been in use for a long time. This is an important reason for choosing these books. Another reason is that they are used at my university, and, in particular regarding the book by Kreyzig, that I know the book well. In addition, I have studied the presentation in online lectures presented by Lars Lundheim in the course Design and Analysis of Electronic Systems II at NTNU (Lundheim, 2019). To complement the presentation in the book by Kreyzig I have looked at the presentation in another book of a similar nature (O’Neil, 2018). The resources mentioned above are the main sources for characterising the praxeologies $\mathscr{P}_{B M}$ and $\mathscr{P}_{E}$. I will emphasise that although the book by Kreyzig carries the word 'engineering' in its title, it is clearly a mathematical text, however with examples showing applications in engineering, but with no particular branch of engineering being foreseen. In the Preface of the book is written: "It is intended to introduce students of engineering, physics, mathematics, computer science and related fields to those areas of applied mathematics that are most relevant for solving practical problems" (Kreyzig, 2011, p. vii). This shows that the book has a wide scope, and I see it as meant to give students a foundation in mathematics, so that they can use mathematics in engineering and science courses later. The book by O'Neil expresses a similar purpose by focusing on providing mathematical topics "needed in the study and practice of engineering" (O’Neil, 2018, p. xv). I therefore see these books as relevant representatives of $\mathscr{P}_{B M}$ with the understanding that $B M$ means basic mathematics for engineering students. For $\mathscr{P}_{A M}$ I have consulted several more advanced expositions of Fourier series, the most important source being the book Fourier Series and Integrals by Dym and McKean (1972), but I have also used the classical text by Rudin (1976). These books, although old, are still used as reference books in advanced courses in analysis. Certainly, the range of books could have been made much wider, and there are books that could be said to lie between the books chosen, regarding the degree of rigour. 
I have performed a detailed analysis of the introduction to Fourier series in Kreyzig's (2011) book (Chapter 11) as well as the introduction to the same topic in the book by Nilsson and Riedel (2011) (Chapter 16) and the section on Fourier series in the module Spectrum of periodic signals in the lectures by Lundheim (2019). Based on data from these sources, complemented with data from texts on more advanced mathematics, I aim to answer the question: What characterises the praxeologies connected to Fourier series in mathematics and in signal theory? I will also aim at identifying the generating questions $Q$ in the institutions under consideration. To get a better understanding of the background for the generating questions and to put the topic in perspective, I find it relevant to also look at the origin of Fourier series. In the next chapter I will give a brief overview of the history of Fourier series.

\section{History of Fourier Series}

In the eighteenth century the concept of function was not yet well established. For example, Euler, in his Introductio in analysis infinitorum from 1748, restricted a function "of a variable quantity" to be "an analytical expression composed in any manner from that variable quantity and numbers or constant quantities" (Kleiner, 1989, p. 3; Rüthing, 1984, p. 72), without further defining the term "analytical expression"”. At about the same time as Euler published his work, the interest in the so-called Vibrating-String Problem developed, and this problem also involved a discussion of the meaning of function (Edwards, 1979, p. 301; Kleiner, 1989, p. 4). Kleiner refers to the following as "an 'article of faith' of $18^{\text {th }}$ century mathematics: If two analytic expressions agree on an interval, they agree everywhere” (1989, p. 4). In 1747, d'Alembert presented a solution to the Vibrating-String Problem but Euler did not believe that d'Alembert's solution was the most general. Bernoulli, in 1753, presented a solution in terms of a trigonometric series but this solution was contested by several contemporaries because it did not comply with "the article of faith" (Kleiner, 1989, pp. 5-6). Kleiner, quoting Ravetz, refers to the debate around the Vibrating-String Problem as a debate "between d'Alembert's mathematical world, Bernoulli's physical world, and Euler's 'no-man's land' between the two" (Kleiner, 1989, p. 7). In the language of ATD one may say that this debate is an example of different praxeologies involved in finding the answer to the same generating question, how to describe the motion of a vibrating string?

Although Bernoulli gave the solution of the Vibrating-String Problem in terms of a trigonometric series, it was only through the work of Fourier that a comprehensive general theory of trigonometric series was developed. Fourier published his book Théorie Analytique de la Chaleur (The Analytical Theory of Heat) in 1822, having submitted his work to the Paris Academy of Sciences already in 1807 (Kleiner, 1989, p. 289). In this book he studied a steady-state temperature function $u(x, y)$ on a region $0 \leq x \leq \pi, y \geq 0$, satisfying the partial differential equation, with boundary conditions, known as the Heat Equation (Edwards, 1979, p. 304). Fourier claims that any function on an interval $(-l, l)$ can be represented on this interval by a trigonometric series. Fourier also gave explicit formulas for the coefficients of the series, in terms of integrals. These formulas correspond exactly to the

\footnotetext{
${ }^{1}$ In Euler's original words, "expressio analytica” (Rüthing, 1984, p. 72).
} 
formulas that are presented in modern books, see (3). Issues that he needed to deal with were to show that the coefficients could be calculated for any function and that any function could be represented by its series in $(-l, l)$. Kleiner writes that "Fourier accomplished all this using mathematical reasoning that would be clearly unacceptable to us today" $(1989$, p. 8). Using his method, Fourier obtained the series representation of a rectangular wave as

$$
\cos x-\frac{1}{3} \cos 3 x+\frac{1}{5} \cos 5 x-\frac{1}{7} \cos 7 x+\ldots=\sum_{k=0}^{\infty}(-1)^{k} \frac{1}{2 k+1} \cos (2 k+1) x .
$$

This series has the value $\frac{\pi}{4}$ for $-\frac{\pi}{2}<x<\frac{\pi}{2}$ and the value $-\frac{\pi}{4}$ for $\frac{\pi}{2}<x<\frac{3 \pi}{2}$, and then continues periodically with period $2 \pi$. For odd multiples of $\frac{\pi}{2}$, the series has the value zero, whereas the function to which it converges is not defined for these $x$-values. Hence, this is an example of a series composed of continuous functions converging to a function which is not continuous. According to Katz (1998, p. 722), Fourier represented the series as a square wave, also drawing the vertical line segments at the odd multiples of $\frac{\pi}{2}$ joining the horizontal line segments at distance $\frac{\pi}{4}$ above and below the horizontal axis. Even though this curve would be continuous, in a naïve sense of the word, it would not represent a function, since values at odd multiples of $\frac{\pi}{2}$ would not be uniquely defined. This did not seem to worry Fourier since he mainly was interested in the physical problem, the praxis block, and not so much in the rigorous mathematics, the logos block (Katz, 1998). The work of Fourier had significant implications for further development of the concept of function, and for the concepts of convergence and continuity (Edwards, 1979; Kleiner, 1989, 2012). To illustrate the phenomenon described above, see Fig. 1 which contains two plots, made using Maple, of partial sums of the series (1), for $n=5$ (left) and $n=20$ (right).

\section{A Praxeological Analysis of Modern Expositions of Fourier Series}

In this chapter I will look at Fourier series both from the perspective of mathematics and from the perspective of signal theory to answer the question: What characterises the praxeologies connected to Fourier series in mathematics and in signal theory? First, I will look at the presentation in basic mathematics, then in signal theory, and at the end I will turn to more advanced mathematics to get a wider perspective.

\section{Fourier Series in Basic Mathematics}

The chapter on Fourier analysis in Kreyzig's (2011) book, Chapter 11, starts by describing Fourier series as infinite series representing general periodic functions in terms of sines and cosines. It is claimed that therefore "Fourier series are of greatest importance to the engineer and applied mathematician" (Kreyzig, 2011, p. 474). Applications, for example to forced oscillations are mentioned. The motivation for introducing Fourier series seems to be to solve ordinary differential equations for forced oscillations and to approximate periodic functions. Later, applications to partial differential equations are presented, much in line with the motivation behind Fourier's original work. 


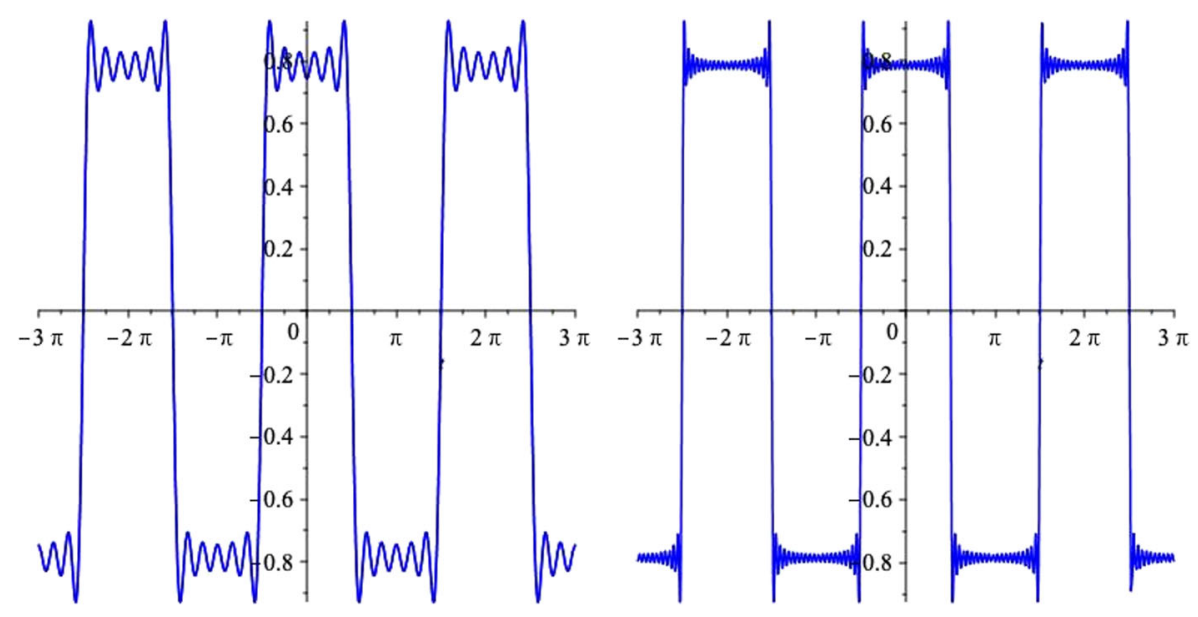

Fig. 1 Partial sums of Fourier series of a rectangular wave $(n=5$, left, and $n=20$, right)

The aim of the chapter is said to be to represent functions of period $2 \pi$ in terms of the sine and cosine functions. Based on this, I take the question, $Q_{B M}$, of the praxeology $\mathscr{P}_{B M}=\left[T_{B M}, \tau_{B M}, \theta_{B M}, \Theta_{B M}\right]$ to be "how can periodic functions be represented in terms of trigonometric functions?" I will now turn to a discussion of the components $T_{B M}$, $\tau_{B M}$, and $\theta_{B M}$ of $\mathscr{P}_{B M}$. I postpone the discussion of $\Theta_{B M}$. The first worked example of a Fourier series in Kreyzig's book (2011, pp. 477-478) is a periodic rectangular wave, given by

$$
f(x)=\left\{\begin{array}{c}
-k, \quad-\pi<x<0 \\
k, \quad 0<x<\pi
\end{array} \text { and } f(x+2 \pi)=f(x)\right.
$$

Nothing is said about the number $k$, but for the example to be of any value, $k$ must be assumed to be different from zero.

In the exercise section after section 11.1, there are several exercises of the type "find the Fourier series of a given $2 \pi$-periodic function and graph partial sums of this series". Based on this, I take the task, $T_{B M}$, to be to find the Fourier series of a given $2 \pi$-periodic function and graph partial sums of this series. The techniques, $\tau_{B M}$, can be seen in the computations of the Fourier coefficients of the function in (2). Before these computations are performed the standard formulas for the Fourier coefficients, as Fourier also had them, are presented, without proof (Kreyzig, 2011, p. 476):

$$
\begin{gathered}
a_{0}=\frac{1}{2 \pi} \int_{-\pi}^{\pi} f(x) d x \\
a_{n}=\frac{1}{\pi} \int_{-\pi}^{\pi} f(x) \cos (n x) d x, \quad n=1,2, \ldots \\
b_{n}=\frac{1}{\pi} \int_{-\pi}^{\pi} f(x) \sin (n x) d x, \quad n=1,2, \ldots
\end{gathered}
$$


These are then used on the function (2) to compute the coefficients, using standard integration methods based on known facts about trigonometric functions.

There are however, several issues that come up when performing these techniques, having to do with why the various operations may be performed. These issues are part of the technology, $\theta_{B M}$. In developing the formulas (3), the infinite series

$$
a_{0}+\sum_{n=1}^{\infty}\left(a_{n} \cos n x+b_{n} \sin n x\right),
$$

after termwise multiplication with suitably chosen functions, is integrated term by term, without justifying why this is allowed. It is just assumed that it is allowed. ${ }^{2}$ There is also no argument to justify whether the series converges. It is just claimed that if it converges, then its sum will have period $2 \pi$, like each of the terms has. This shows that the technology $\theta_{B M}$ is insufficient to justify the techniques, $\tau_{B M}$. In the integration process, properties of trigonometric functions such as $\int_{-\pi}^{\pi} \sin (m x) \sin (n x) d x=0, \quad m \neq n$ are used. This, and similar properties for the product of cosines and the product of sine and cosine, can be verified directly by computation. Kreyzig (2011, p. 479) refers to this property as orthogonality of the trigonometric system and mentions that this amounts to a generalisation of inner product as this is known from vectors. However, the concept of orthogonality is not really needed at this point, since only trigonometric functions are involved, and then the orthogonality follows directly from basic properties of these functions.

Here two fundamental questions arise: Which functions can be represented in a series of the form (4), and under which conditions, and to what, does the series converge? Kreyzig (2011, p. 480) presents a theorem (Theorem 2) where a sufficient condition for such a representation to exist is given: $f$ is assumed to be piecewise continuous on the interval $-\pi \leq$ $x \leq \pi$, and have a left- and a right-hand-side derivative at each point in the interval. Under these conditions, the theorem states that the series converges to $f$, except at discontinuities, where it converges to the average of the left- and right-hand-side limit of $f(x)$. In the proof of the theorem even further restrictions are made: "We prove convergence, but only for a continuous function $f(x)$ having continuous first and second derivatives. And we do not prove that the sum of the series is $f(x)$ because these proofs are much more advanced" (Kreyzig, 2011, p. 481). The main step in the proof is that, because of the assumptions on continuity, it follows that the coefficients $a_{n}$ and $b_{n}$ will in absolute value be bounded by a constant over $n^{2}$, and then the convergence follows from the convergence of the series $\sum_{n=1}^{\infty} \frac{1}{n^{2}}$. It should be noted that the function (2) is not continuous, and hence certainly not differentiable on $\mathbb{R}$, so the proof that is presented, does not even cover the example that I take to be the fundamental motivating example for the concept of Fourier series. The presented tasks, and the techniques to solve them are therefore not covered by the technology that is presented.

There are many books available with the title "Advanced Engineering Mathematics”, and I have also looked at another book with this title (O’Neil, 2018). In this book, the starting point for Fourier series is a bit different that in Kreyzig's (2011) book. O’Neil starts with differential equations known as Sturm-Liouville problems, and discusses eigenvalues and eigenfunctions of such problems. Fourier series are

\footnotetext{
$\overline{2}$ The concept uniform convergence is briefly mentioned on page 481, with reference to a later chapter in the book.
} 
introduced as special eigenfunction expansions. As for the question of convergence, O'Neil states that the Fourier series of a function $f(x)$ that is piecewise smooth on an interval $[-L, L]$ converges on the open interval $-L<x<L$ to $\frac{1}{2}(f(x-)+f(x+))$ (mean value of left- and right-hand-side limits), and at the endpoints to the one-sided limits. However, there is no proof of this result, just a remark that for now "we have enough background to use Fourier expansions to solve partial differential equations" (O'Neil, 2018 , p. 134). So also here, a substantial technology to cover the tasks and techniques is lacking.

\section{Fourier Series in Signal Theory}

In this section I will give a brief exposition of an introduction to Fourier series in signal theory, following two sources; the book by Nilsson and Riedel (2011) and the video lectures by Lundheim (2019). Nilsson and Riedel start the chapter on Fourier series with what they refer to as a practical perspective, bandpass and bandreject filters. They then state that "[i]n this chapter we will learn that any periodic signal can be represented as a sum of sinusoids, where the frequencies of the sinusoids are comprised of the frequency of the periodic signal and integer multiples of that frequency" (Nilsson \& Riedel, 2011, p. 627). Further, they motivate periodic signals by saying that such signals can be used to test the quality of a bandpass or a bandreject filter, and they mention that periodic signals are important in power generators, and also in nonelectrical systems. After an introduction with motivation for studying periodic signals, they introduce Fourier series with reference to Fourier and his investigation of heat-flow problems. The representation is given as

$$
f(t)=a_{v}+\sum_{n=1}^{\infty} a_{n} \cos n \omega_{0} t+b_{n} \sin n \omega_{0} t
$$

where $\omega_{0}=2 \pi / T$ is called the fundamental frequency of the function $f$ with period $T$ (Nilsson \& Riedel, 2011, p. 629). Integer multiples of $\omega_{0}$ are called the harmonic frequencies. The formulas for the Fourier coefficients are here given in the same way as in Kreyzig (2011), except for small differences in notation. Nilsson and Riedel (2011, p. 630) write (compare 3)

$$
\begin{gathered}
a_{v}=\frac{1}{T} \int_{t_{0}}^{t_{0}+T} f(t) d t \\
a_{k}=\frac{2}{T} \int_{t_{0}}^{t_{0}+T} f(t) \cos k \omega_{0} t d t \\
b_{k}=\frac{2}{T} \int_{t_{0}}^{t_{0}+T} f(t) \sin k \omega_{0} t d t
\end{gathered}
$$

It is made a point of the fact that as long as the function is integrated over a whole period, it does not matter where the starting point is. Lundheim (2019) makes the same point by just writing $T$ below the integral sign. Note also that instead of $a_{0}$ in (3) the constant term in (6) is denoted by $a_{v}$. The notation $a_{v}$ is motivated by saying that the value of $a_{v}$ is the average value of $f(t)$ (Nilsson \& Riedel, 2011, p. 630). This is completely in line with the mathematical definition of the average value of a function over an interval, as the integral of the function over the interval divided by the length of 
the interval. No such interpretation of the constant term is given by Kreyzig (2011). In signal theory, the constant term is also often referred to as the DC (direct current) component of the signal, linking the role of the constant term even closer to the physical reality. Nilsson and Riedel give a list of sufficient conditions, called Dirichlet's conditions, for a periodic function to have a convergent Fourier series and then state that "[a]ny periodic function generated by a physically realizable source satisfies Dirichlet's conditions" (Nilsson \& Riedel, 2011, p. 629). The formulas for the Fourier coefficients are justified by direct integration, very much like it was done by Kreyzig (2011), using elementary properties of trigonometric functions. A difference is that whereas Kreyzig made a note that he assumed that termwise integration was allowed, Nilsson and Riedel do not even mention that there might be problems with performing termwise integration. The first worked example in the book by Nilsson and Riedel is to find the Fourier series for a periodic voltage given by the function $v(t)=\frac{V_{m}}{T} t$ on the interval $0<t<T$, and having period $T$. This is worked out using the formulas (6).

For the praxeology $\mathscr{P}_{E}=\left[T_{E}, \tau_{E}, \theta_{E}, \Theta_{E}\right]$, it can so far be noted that parts of the technique are very much like in $\mathscr{\rho}_{B M}$, based on properties of trigonometric functions. The main differences are in notation, which I also take to be part of the technique. As for the technology, no attempts are made in $\mathscr{P}_{E}$ to prove that a function with certain given properties has a convergent Fourier series. Only some sufficient conditions (the Dirichlet conditions) are stated, without proof. As observed in the previous section, in $\mathscr{P}_{B M}$ there is a proof, but the proof assumes much stronger conditions than the functions that, according to the praxeology, are of most interest, satisfy.

From the introduction of Fourier series by Nilsson and Riedel (2011), it may seem that the generating question is how to represent periodic functions in a series built up of sines and cosines. However, from the section Practical Perspective (Nilsson \& Riedel, 2011, p. 627), one gets the impression that there is more behind, namely that this topic will be useful in order to work with bandpass and bandreject filters. A hint towards applications is also given by referring to $\omega_{0}$ as the frequency of the signal. In the lectures by Lundheim (2019) the generating question is more directly connected to signal theory, as his motivation for Fourier series is to find the spectrum of a periodic signal. He presents the Fourier series in its complex form,

$$
x(t)=\sum_{-\infty}^{\infty} c_{k} e^{j \omega_{k} t}, \quad c_{k}=\frac{1}{T} \int_{T} x(t) e^{-j \omega_{k} t} d t, \quad \omega_{k}=\frac{2 \pi}{T} k .
$$

In accordance with the usage in electrical engineering, the imaginary unit is denoted by $j$ instead of $i$ as is common in mathematics. After presenting the representation (7) he gives an example (with $a=1 / 2$ ) of the square wave represented by

$$
x(t)=\left\{\begin{array}{c}
1 \text { for } \quad|t|<a \frac{T}{2} \\
0 \text { for } a \frac{T}{2}<|t|<\frac{T}{2}
\end{array}\right.
$$

extended periodically to have period $T$. Now, using the formula for $c_{k}$ from (7) Lundheim obtains (with $a=1 / 2$ ) 


$$
c_{k}=\frac{1}{2} \frac{\sin (\pi k / 2)}{\pi k / 2}=\frac{1}{2} \operatorname{sinc}(k / 2),
$$

where $\operatorname{sinc}(x)$ is defined as $\operatorname{sinc}(x)=\frac{\sin (\pi x)}{\pi x}$. At this point he presents a graph of the function $\frac{1}{2} \operatorname{sinc}(k / 2)$ and uses this graph to indicate the values of $c_{k}$ depending on whether $k$ is even or odd. Combining terms for $k$ and $-k$, and pulling out the term for $k=$ 0 , Lundheim writes the series in (7) as

$$
x(t)=c_{0}+\sum_{k=1}^{\infty} c_{k} e^{j \omega_{k} t}+c_{-k} e^{-j \omega_{k} t}
$$

Now, some arguments follow: First, Lundheim states that "it can be shown" that since $x(t)$ in (8) is an even function, the coefficients $c_{k}$ are real. Further, he observes that since $\operatorname{sinc}(x)$ is even, $c_{k}=c_{-k}$. And finally, he states that "it can be shown" that since $x(t)$ is real, it follows that $c_{k}=c_{-k}^{*}$, (* here denotes the complex conjugate). In this reasoning, part of the justification is hidden in the phrase "it can be shown". On the basis of this, he obtains

$$
c_{k} e^{j \omega_{k} t}+c_{-k} e^{-j \omega_{k} t}=c_{k} e^{j \omega_{k} t}+c_{k}^{*} e^{-j \omega_{k} t}=2 \operatorname{Re}\left\{c_{k} e^{j \omega_{k} t}\right\}=2\left|c_{k}\right| \cos \left(\omega_{k} t+\angle c_{k}\right),
$$

where $\angle c_{k}$ is called the phase angle of $c_{k}$, from then on denoted $\varphi_{k}$. This gives the representation

$$
x(t)=c_{0}+2 \sum_{k=1}^{\infty}\left|c_{k}\right| \cos \left(\omega_{k} t+\varphi_{k}\right)
$$

Since $c_{k}$ is real, $\varphi_{k}$ is either 0 or $\pi$. Lundheim refers to $\left|c_{k}\right|$ as the amplitude of the signal and $\omega_{k}$ as the frequencies. Hence, all the parameters in the representation of the Fourier series are interpreted within $\mathscr{P}_{E}$.

This way of seeing the Fourier series expansion is motivated by the generating question about finding the spectrum of a periodic signal. Fig. 2 shows the amplitude spectrum, $\left|c_{k}\right|$, for the function $x(t)$ in (8) with $a=0.2$ and $T=1$, for $k=0,1, \ldots, 20$. Figure 3 shows the actual values of the Fourier coefficients $c_{k}$ for $k=0,1, \ldots, 20$. They follow the graph of the function $s(x)=\frac{\sin (\pi a x)}{\pi a x}$ and take both positive $\left(\varphi_{k}=0\right)$ and negative values $\left(\varphi_{k}=\pi\right)$.

In the praxeologies that I have looked at so far, the question of whether a given function has a Fourier series expansion is not dealt with in great precision. Kreyzig (2011, p. 480) states a theorem in which some sufficient conditions are given. In the 
proof, however, he makes further assumptions, not even fulfilled by the functions appearing in most of the examples and exercises. Therefore, the technology $\theta_{B M}$ can be said to be insufficient for the tasks and techniques in the praxeology. In $\mathscr{P}_{E}$ the situation is similar. One can even say that here the technology is weaker, as Nilsson and Riedel (2011) just claim that the Dirichlet conditions are sufficient condition for a function to be expressed in a convergent Fourier series. Lundheim (2019) leaves the theoretical issues to a large extent to the subject of mathematics. It can therefore be said that in $\mathscr{P}_{E}$ the logos block comes from some unspecified mathematical praxeology $\mathscr{P}_{M}$.

\section{Fourier Series in Advanced Mathematics}

In this section I will dig a little deeper into both the theory and the technology to try to identify some of the challenges that are involved when aiming for a higher level of precision. As I have indicated earlier, Fourier's own development of the theory was also not very precise, and he was aware of that. The main sources for my analysis here are two books, Dym and McKean (1972) and Rudin (1976). For lack of a better word, I will refer to the praxeology represented by these two books as a praxeology of advanced mathematics, $\mathscr{P}_{A M}$.

Dym and McKean write that the key to further progress regarding Fourier series was provided by the Lebesgue integral, and that "[ $t$ ]he proper setting for Fourier series turned out to be the class of 'Lebesgue measurable' functions $f$ of period 1" (Dym \& McKean, 1972, p. 2). Later, Dym and McKean introduce the class $L^{2}(Q)$, the class of all complex (measurable) functions $f$ on a subinterval $Q$ of the real numbers. They write that this is the class of functions with which Fourier series are most naturally associated (Dym \& McKean, 1972, pp. 12-13). For such functions, the inner product is defined as $\langle f, g\rangle=\int_{Q} f \bar{g} d \mu$, where $\mu$ is the Lebesgue measure, and the norm of a Lebesgue

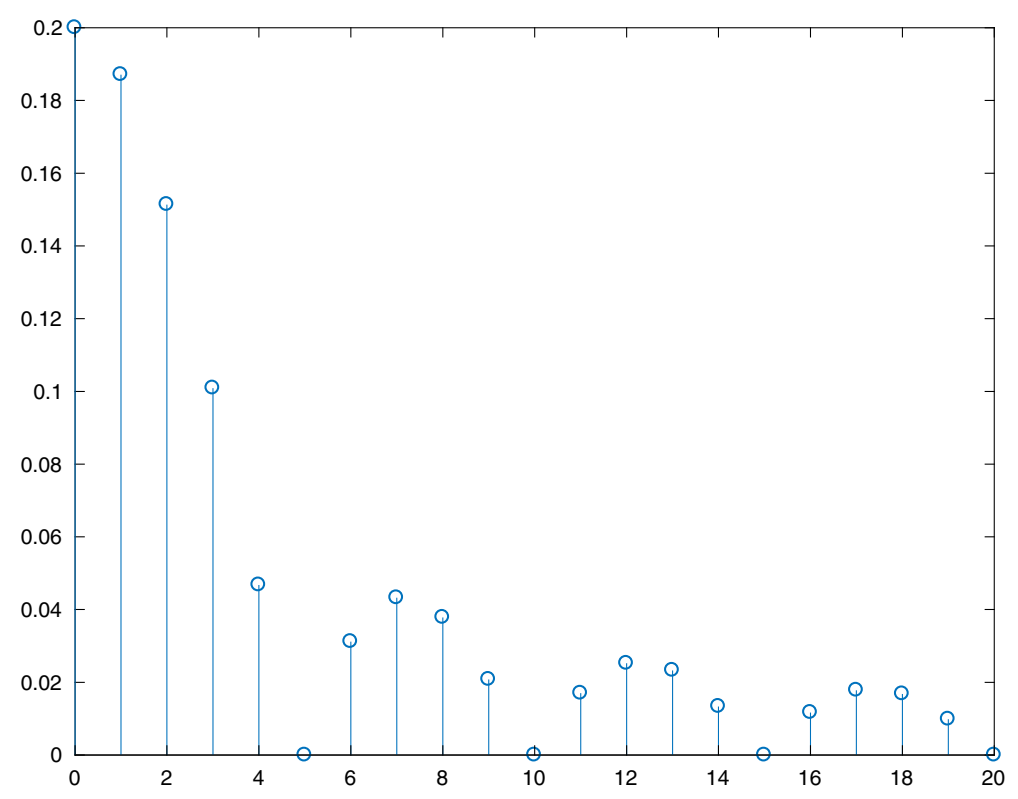

Fig. 2 The amplitude spectrum 


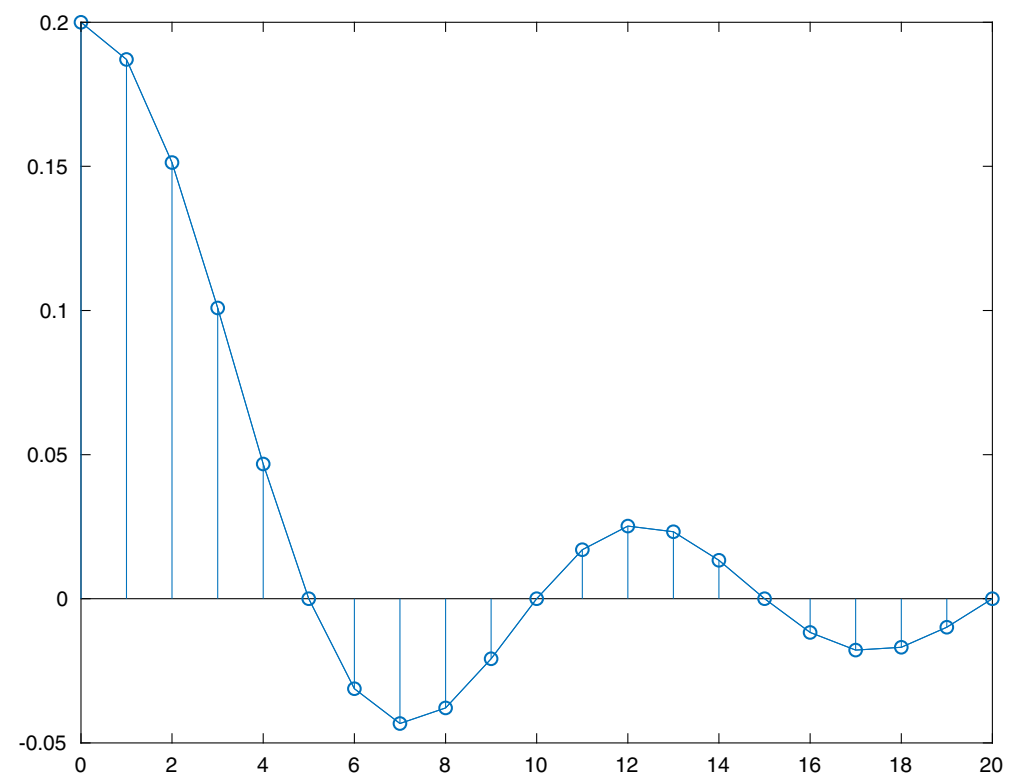

Fig. 3 The Fourier coefficients

integrable function is defined as $\|f\|=\langle f, f\rangle^{\frac{1}{2}}<\infty$. A family of functions $\left\{\varphi_{k}\right\}$ is called orthonormal if $\left\|\varphi_{k}\right\|=1$ and $\left\langle\varphi_{i}, \varphi_{j}\right\rangle=0$ for $i \neq j$. The Fourier coefficients are defined as $\widehat{f}(k)=\int_{Q} f \bar{\varphi}_{k} d \mu$, where $\left\{\varphi_{k}\right\}$ is an orthonormal family. The formal Fourier series is formed as the expansion $\sum_{k=1}^{\infty} \widehat{f}(k) \varphi_{k}$ and a question is then whether this series converges, and if so, what does it converge to? Two results are important in this context:

1. For any $f$ in $L^{2}(Q),\left\{\varphi_{k}\right\}$ an orthonormal family, and any sequence of complex numbers $\left\{c_{k}\right\},\left\|f-\sum_{k=1}^{n} \widehat{f}(k) \varphi_{k}\right\| \leq\left\|f-\sum_{k=1}^{n} c_{k} \varphi_{k}\right\|$, where $\widehat{f}(k)$ are the Fourier coefficients with respect to $\left\{\varphi_{k}\right\}$. (Dym \& McKean, 1972, p. 23; Rudin, 1976, p. 187)

2. Let $\left\{\varphi_{k}\right\}$ be an orthonormal family. If $\sum_{k=1}^{\infty}\left|c_{k}\right|^{2}<\infty$ then there exists a Lebesgue measurable function $f$ such that $\left\|f-\sum_{k=1}^{n} c_{k} \varphi_{k}\right\| \rightarrow 0$ when $n \rightarrow \infty$, and $f$ can be represented by the series $\sum_{k=1}^{\infty} c_{k} \varphi_{k}$. (Rudin, 1976, p. 330)

The result 1. states that the partial sums of the Fourier series give the best approximation in $L^{2}$ norm, and from 2. it follows, using the orthonormality of $\left\{\varphi_{k}\right\}$, that $c_{k}=\int_{Q} f \bar{\varphi}_{k} d \mu$. The result 2. is often referred to as the Riesz-Fischer Theorem and is taken to be a fundamental result in the theory of Fourier series. Dym and McKean express the Riesz-Fischer theorem as saying that there is a 1-1 map of the space of Lebesgue measurable functions onto the space of square summable sequences of numbers, or in other words $L^{2}(Q)$ and $L^{2}\left(Z^{+}\right)$are isomorphic (Dym \& McKean, 1972, p. 3 and p. 26). As the first example of an orthonormal family, Dym and McKean present the functions $e_{k}(x)=e^{2 \pi i k x}$ on the 
interval $[0,1]$. The crucial point here is that the series expansion uses an orthonormal family as a basis. As mentioned in the subchapter on basic mathematics, also Kreyzig (2011) introduces the concept of orthogonality of functions, but the concept has no real significance in his exposition since he only considers trigonometric functions, and then the orthogonality follows trivially by integration. In the praxeology $\mathscr{P}_{A M}$ however, orthogonality (orthonormality) becomes a crucial concept. Lundheim (2019) uses the complex form of the Fourier series but since the complex exponential can be written in terms of sine and cosine functions, the orthogonality also here is granted.

In the praxeology $\mathscr{P}_{A M}$ the Fourier series of a function $f$ is taken as a formal construct and an important question is to characterise the functions $f$ for which this series converges to $f$. Finding these conditions can be seen as the first task in this context. Dym and McKean (1972), after having established the fundamental results, go on to applications of Fourier series, for example to partial differential equations, and this can also be seen as tasks. I will not go into details about the proofs of the results quoted above, but I will end this section with the remark that a significant difference between the praxeologies $\mathscr{P}_{B M}$ and $\mathscr{P}_{E}$ compared to $\mathscr{P}_{A M}$ is that the theory, $\Theta$, has changed. In $\mathscr{P}_{B M}$ as well as in $\mathscr{P}_{E}$ the underlying theory is the Riemann integral, but in $\mathscr{P}_{A M}$ the underlying theory is the Lebesgue integral. Therefore, $\Theta_{B M} \neq \Theta_{A M}$.

\section{Comparing the Praxeologies}

In this section I will return to the research question "What characterises the praxeologies connected to Fourier series in mathematics and in signal theory?" and I will summarise the answer to this question from two perspectives. First, I will compare the praxeology in basic mathematics, $\mathscr{P}_{B M}$, to the praxeology in signal theory, $\mathscr{P}_{E}$. Then I will compare the praxeology in basic mathematics, $\mathscr{P}_{B M}$, to the praxeology in advanced mathematics, $\mathscr{P}_{A M}$. I will focus on the main differences between the praxeologies under comparison.

\section{The Praxeologies $\mathscr{P}_{B M}$ and $\mathscr{P}_{E}$}

I will start by discussing the generating questions in the two praxeologies $\mathscr{P}_{B M}$ and $\mathscr{P}_{E}$. The generating question $Q_{B M}$ in $\mathscr{P}_{B M}$ I identified as how to represent and approximate periodic functions by means of trigonometric functions. To do this, Fourier series are introduced, and an important point that is made is that Fourier series approximate noncontinuous functions by means of continuous functions. This is also in line with the initial interest in Fourier series in the nineteenth century. The main task $\left(T_{B M}\right)$ is to find a series representation for a given function. The techniques $\left(\tau_{B M}\right)$ are based on integration, using elementary properties of trigonometric functions $\left(\theta_{B M}\right)$. These techniques give the Fourier coefficients and hence the series. The theory, $\Theta_{B M}$, although not explicitly stated, is the Riemann integral.

Nilsson and Riedel also refer to periodic functions, and they motivate the interest in such functions like this: "One reason is that many electrical sources of practical value generate periodic waveforms" (Nilsson \& Riedel, 2011, p. 626). In Lundheim's (2019) introduction to signal theory, the motivation for introducing Fourier series is more explicitly expressed. He wants to define, in a precise way, the notion of the spectrum of 
the signal, also referred to as the content of the signal since it shows the frequencies that the signal is built up of. Since Lundheim's lectures are closer to the didactic system $S\left(X ; Y_{E} ; \boldsymbol{\vee}_{E}\right)$ that I am investigating than the book by Nilsson and Riedel, I take the generating question in $\mathscr{P}_{E}, Q_{E}$, to be how to find the spectrum of a signal. Figure 4 shows the spectrum of a pure sine wave set to frequency $440 \mathrm{~Hz}$ and Fig. 5 shows the spectrum of a rectangular wave, corresponding to the one shown in Fig. 2 based on a "theoretical" rectangular wave. The pictures are made using a frequency analyser. Such tools are, in addition to computational techniques, used in $\mathscr{P}_{E}$ to obtain the spectrum (Fourier coefficients), and can be seen as part of the techniques. The sine wave in Fig. 4 consists of only one frequency (and some noise) and the rectangular wave in Fig. 5 consists of several frequencies that can be read off from the picture. The height of the peaks for each frequency represents the absolute value of the Fourier coefficients in the Fourier series that makes up the rectangular wave. Both in $\mathscr{P}_{B M}$ and in $\mathscr{P}_{E}$ it is important to find the Fourier series of a function but because the generating question is different in the two praxeologies, the focus in $\mathscr{P}_{B M}$ is on the series and its convergence properties, whereas the focus in $\mathscr{D}_{E}$ is on the coefficients. This indicates a difference in the tasks in the two praxeologies.

For the actual computation of Fourier coefficients, the justification in $\mathscr{P}_{E}\left(\theta_{E}\right)$ is based on well-known properties of trigonometric functions. However, regarding the issue of convergence of the series, only sufficient conditions are given, and this issue is not dealt with in a precise manner in either of the praxeologies. I will therefore claim that there are no significant differences in the technology between these two praxeologies, so $\theta_{B M} \approx \theta_{E}$. Also the theory seems to be the same, $\Theta_{B M}=\Theta_{E}$, although it is not explicitly stated. In the parts on basic mathematics and signal theory, I have described differences in notation between $\mathscr{P}_{B M}$ and $\mathscr{P}_{E}$, which then amount to differences in the techniques. These differences are summarised in Table 1, based on Kreyzig (2011) and Lundheim (2019), respectively. The notation in Nilsson and Riedel (2011) is in fact closer to Kreyzig than to Lundheim, showing differences also within $\mathscr{P}_{E}$.

\section{The Praxeologies $\mathscr{P}_{B M}$ and $\mathscr{P}_{A M}$}

As discussed in the parts on basic mathematics and signal theory, neither of the praxeologies $\mathscr{P}_{B M}$ or $\mathscr{P}_{E}$ gives a complete answer to the question about which functions can be represented in a Fourier series. This question, however, I find to be very important in the praxeology $\mathscr{P}_{A M}$. Rudin writes that "[t] he natural question which now arises is ... whether $f$ is determined by its Fourier series" (Rudin, 1976, p. 186). It may be too simplistic to take this question as the generating question in $\mathscr{P}_{A M}$. I will therefore regard this question as one example of a generating question in $\mathscr{P}_{A M}$. Both in $\mathscr{P}_{B M}$ and $\mathscr{P}_{E}$ the theory, $\Theta_{B M}=\Theta_{E}$, is based on the Riemann integral, but to address $Q_{A M}$, the Lebesgue integral is introduced as the theory, so $\Theta_{B M} \neq \Theta_{A M}$. An important technology in $\mathscr{D}_{A M}$ is that the basis for the Fourier expansion is an orthonormal family. A further justification (see e.g. Rudin, 1976, p. 330) is given in terms of the conditions that the Fourier coefficients should be square summable, $\sum_{n=1}^{\infty}\left|c_{n}\right|^{2}<\infty$. In the part on basic mathematics, I quoted from the proof that Kreyzig (2011) gives for convergence where he shows that the conditions he puts on the function, imply that $\left|a_{n}\right|<\frac{M}{n^{2}}$, where $M$ is a constant (similarly for $\left.\left|b_{n}\right|\right)$. Therefore, the coefficients are square summable (Kreyzig, 2011, p. 481). So square summability is important, but in $\mathscr{P}_{A M}$ 


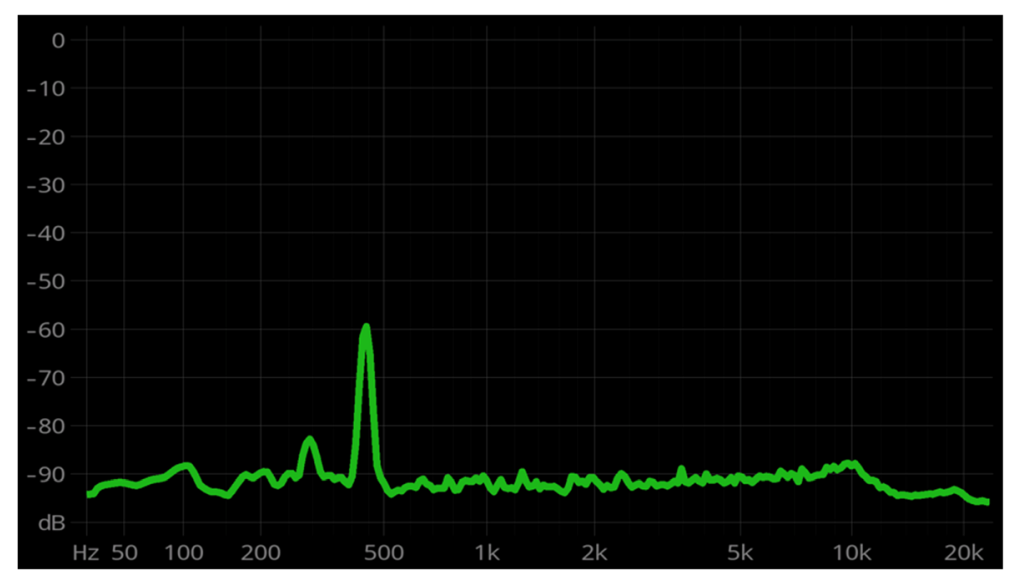

Fig. 4 Spectrum of a pure sine wave

this comes as a condition, whereas in $\mathscr{P}_{B M}$, it comes as a consequence of much too strong conditions on the functions under consideration.

\section{Summary of the Praxeological Analysis}

I will end this chapter by giving an overview of the most important elements that I have discussed in the praxeologies under consideration. I present this overview in Table 2.

\section{Discussion}

The comparison of the praxeologies showed that between $\mathscr{D}_{B M}$ and $\mathscr{D}_{E}$ there is a difference in the generating question, which in $\mathscr{P}_{B M}$ leads to a strong focus on the whole series and its convergence properties, whereas in $\mathscr{P}_{E}$ the focus is more on the Fourier coefficients, representing the spectrum of the signal. It also turned out that the notation was somewhat different in the two praxeologies, meaning that the techniques

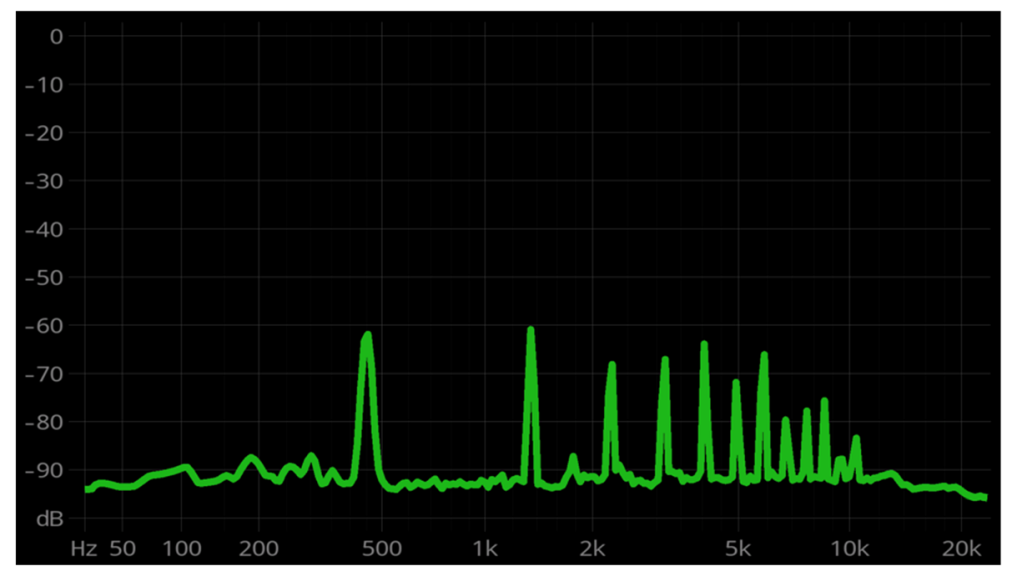

Fig. 5 Spectrum of a rectangular wave 
Table 1 Comparing notation between $\mathscr{\rho}_{\mathrm{BM}}$ and $\mathscr{\rho}_{\mathrm{E}}$

\begin{tabular}{lll}
\hline & $\mathscr{P}_{B M}$ & $\mathscr{P}_{E}$ \\
\hline Imaginary unit & The letter $i$ & The letter $j$ \\
Fourier series representation & $f(x)=a_{0}+\sum_{n=1}^{\infty}\left(a_{n} \cos n x+b_{n} \sin n x\right)$ & $x(t)=\sum_{-\infty}^{\infty} c_{k} e^{j \omega_{k} t}$ \\
& & $x(t)=c_{0}+2 \sum_{k=1}^{\infty}\left|c_{k}\right| \cos \left(\omega_{k} t+\varphi_{k}\right)$ \\
Fourier coefficients & $a_{0}=\frac{1}{2 \pi} \int_{\pi}^{\pi} f(x) d x$ & $c_{k}=\frac{1}{T} \int_{T} x(t) e^{-j \omega_{k} t} d t, \quad \omega_{k}=\frac{2 \pi}{T} k$ \\
& $a_{n}=\frac{1}{\pi} \int_{-\pi}^{\pi} f(x) \cos (n x) d x, \quad n=1,2, \ldots$ & \\
& $b_{n}=\frac{1}{\pi} \int_{-\pi}^{t} f(x) \sin (n x) d x, n=1,2, \ldots$ & \\
\hline
\end{tabular}

at least to some extent, are different. In previous research, many authors have discussed lack of connection between mathematics and engineering regarding relevance of mathematics and also lack of connection regarding mathematical knowledge (Biehler et al., 2015; Carvalho \& Oliveira, 2018; Flegg et al., 2012, Gueudet \& Quéré, 2018; Harris et al., 2015). Alpers (2017) noticed also essential differences in the use of concepts and in notation. This is supported in my investigations.

Much of the previous research has been connected to mechanical engineering (González-Martín \& Hernandes-Gomes, 2017, 2018, 2019a) and the concept of the integral. In this context, an important finding was that the concept of integral was used to define the notions in question, but that the tasks could be solved by simple arguments, not using integration techniques. In the case of signal theory, this is different. I have pointed to differences in the techniques, but these differences are mainly connected to notation. When it comes to actual computation techniques, the situation in mathematics (Kreyzig, 2011; O’Neil, 2018), is pretty much the same as in signal theory (Lundheim, 2019; Nilsson \& Riedel, 2011).

Peters et al. (2017) also used ATD to compare mathematics and signal theory. It seems that their example shows a clearer separation of techniques and technology between mathematics and signal theory than I have observed. Their example is a concrete example from signal theory intended to show that a given envelope detector delivers a signal with certain properties (the task). To solve this task, techniques and technologies both from

Table 2 Overview of the generating questions Q and the four Ts in different praxeologies

\begin{tabular}{|c|c|c|c|}
\hline & $\oint_{B M}$ & $\mathscr{\rho}_{E}$ & $\bigodot_{A M}$ \\
\hline$Q$ & $\begin{array}{l}\text { Represent periodic } \\
\text { functions in a series }\end{array}$ & $\begin{array}{l}\text { Find the spectrum (frequency content) } \\
\text { of a signal }\end{array}$ & $\begin{array}{c}\text { Characterise functions that can be } \\
\text { represented in a Fourier series }\end{array}$ \\
\hline$T$ & $\begin{array}{l}\text { Compute Fourier } \\
\text { coefficients to get the } \\
\text { Fourier series }\end{array}$ & Compute Fourier coefficients & $\begin{array}{l}\text { Find conditions for representation } \\
\text { and convergence of Fourier } \\
\text { series }\end{array}$ \\
\hline$\tau$ & $\begin{array}{l}\text { Computations using } \\
\text { trigonometric functions }\end{array}$ & $\begin{array}{l}\text { Computations using trigonometric } \\
\text { functions. Frequency analyser }\end{array}$ & $\begin{array}{l}\text { Inner products. Norm. } \\
\text { Orthonormal families }\end{array}$ \\
\hline$\theta$ & $\begin{array}{l}\text { Properties of trigonometric } \\
\text { functions }\end{array}$ & $\begin{array}{l}\text { Properties of trigonometric functions. } \\
\text { Observations from the frequency } \\
\text { analyser }\end{array}$ & Properties of the space $L^{2}(Q)$ \\
\hline$\Theta$ & The Riemann integral & The Riemann integral & The Lebesgue integral \\
\hline
\end{tabular}


mathematics and engineering are applied. My example is on a more basic level in the engineering course. A hypothesis could then be, that the praxeologies $\mathscr{P}_{B M}$ and $\mathscr{P}_{E}$ will differ more as the engineering course progresses. Therefore, it would be of interest to follow the development of an engineering course over a longer period of time.

Regarding the mathematical praxeologies, $\mathscr{P}_{B M}$ and $\mathscr{P}_{A M}$, it is worth noticing that there is a significant difference in the theory, $\Theta_{B M} \neq \Theta_{A M}$, and this difference is based on the development from the Riemann to the Lebesgue integral. This difference also echoes the historical development of Fourier series, in the sense that when Fourier series first were introduced, the theory of the Lebesgue integral was not yet developed, which could explain why Fourier and his contemporaries were not in the position to establish a rigorous foundation for their results.

Acknowledgments The author would like to thank the reviewers and the editor for very constructive and helpful comments.

Funding Open access funding provided by NTNU Norwegian University of Science and Technology (incl St. Olavs Hospital - Trondheim University Hospital).

Open Access This article is licensed under a Creative Commons Attribution 4.0 International License, which permits use, sharing, adaptation, distribution and reproduction in any medium or format, as long as you give appropriate credit to the original author(s) and the source, provide a link to the Creative Commons licence, and indicate if changes were made. The images or other third party material in this article are included in the article's Creative Commons licence, unless indicated otherwise in a credit line to the material. If material is not included in the article's Creative Commons licence and your intended use is not permitted by statutory regulation or exceeds the permitted use, you will need to obtain permission directly from the copyright holder. To view a copy of this licence, visit http://creativecommons.org/licenses/by/4.0/.

\section{References}

Alpers, B. (2008). The mathematical expertise of mechanical engineers - the case of machine element dimensioning. In B. Alpers, S. Hibberd, D. Lawson, L. Mustoe, \& C. Robinson (Eds.), Proceedings of 14 SEFI (MWG) conference, Loughborough, 6-9 April 2008. Aalen, Germany: European Society for Engineering Education (SEFI) Retrieved from http://sefi.htw-aalen.de/Seminars/Loughborough2008/ mee2008/pages/proceedings.html.

Alpers, B. (2017). Differences between the usage of mathematical concepts in engineering statics and engineering mathematics education. In R. Göller, R. Biehler, R. Hochmuth, \& H.-G. Rück (Eds.), Didactics of mathematics in higher education as a scientific discipline. Conference proceedings (pp. 137-141). Kassel, Germany: University of Kassel.

Alpers, B. (2020). Mathematics as a service subject at the tertiary level. A state-of-the-art report for the Mathematics Interest Group. Brussel, Belgium: European Society for Engineering Education (SEFI).

Bajpaj, A. C. (1985). The role of mathematics in engineering education: A mathematician's view. International Journal of Mathematical Education in Science and Technology, 16(3), 417-430.

Biehler, R., Kortemeyer, J., \& Schaper, N. (2015). Conceptualizing and studying students' processes of solving typical problems in introductory engineering courses requiring mathematical competences. In $\mathrm{K}$. Krainer \& N. Vondrová (Eds.), Proceedings of the ninth congress of the European society for research in mathematics education (pp. 2060-2066). Prague, Czech Republic: Charles University and ERME.

Bosch, M., \& Gascón, J. (2014). Introduction to the anthropological theory of the didactic (ATD). In A. Bikner-Ahsbahs \& S. Prediger (Eds.), Networking of theories as a research practice in mathematics education (pp. 67-83). Cham, Switzerland: Springer.

Carvalho, P., \& Oliveira, P. (2018). Mathematics or mathematics for engineering? In Proceedings from 2018 $3 r d$ international conference of the Portuguese society for engineering education (CISPEE). Retrieved from https://ieeexplore.ieee.org/document/8593463/authors\#authors 
Chevallard, Y. (2006). Steps towards a new epistemology in mathematics education. In M. Bosch (Ed.), Proceedings of the fourth congress of the European society for research in mathematics education (pp. 21-30). Barcelona, Spain: FUNDEMI-IQS and ERME.

Dym, H., \& McKean, H. P. (1972). Fourier series and integrals. San Diego, CA: Academic Press.

Edwards, C. H. (1979). The historical development of the calculus. New York: Springer.

Enelund, M., Larsson, S., \& Malmqvist, J. (2011). Integration of a computational mathematics education in the mechanical engineering curriculum. In P. M. Hussmann (Ed.), Conference proceedings 7th international CDIO conference, technical university of Denmark, 20th - 23th June 2011 (pp. 996-1012). Lyngby, Denmark: Technical University of Denmark.

Flegg, J., Mallet, D., \& Lupton, M. (2012). Students' perceptions of the relevance of mathematics in engineering. International Journal of Mathematics Education in Science and Technology, 43(6), 717-732.

González-Martín, A. S., \& Hernandes-Gomes, G. (2017). How are calculus notions being used in engineering? An example with integrals and bending moments. In T. Dooley \& G. Gueudet (Eds.), Proceedings of the tenth congress of the European society for research in mathematics education (CERME 10) (pp. 2073-2080). Dublin, Ireland: DCU Institute of Education \& ERME.

González-Martín, A. S., \& Hernandes-Gomes, G. (2018). The use of integrals in mechanics of materials textbooks for engineering students: The case of the first moment of an area. In V. Durand-Guerrier, R. Hochmuth, S. Goodchild, \& N. M. Hogstad (Eds.), Proceedings of INDRUM 2018. Second conference of the international network for didactic research in university mathematics (pp. 115-124). Kristiansand, Norway: University of Agder and INDRUM.

González-Martín, A. S., \& Hernandes-Gomes, G. (2019a). What happens after calculus? Examples of the use of integrals in engineering: The case of electromagnetism. In J. Monaghan, E. Nardi, \& T. Dreyfus (Eds.), Calculus in upper secondary and beginning university mathematics - Conference proceedings (pp. 99102). Kristiansand, Norway: MatRIC.

González-Martín, A. S., \& Hernandes-Gomes, G. (2019b). How engineers use integrals: The cases of mechanics of materials and electromagnetism. In M. Graven, H. Venkat, A. Essien, \& P. Vale (Eds.), Proceedings of the 43rd conference of the international group for the psychology of mathematics education (Vol. 2, pp. 280-287). Pretoria, South Africa: PME.

Gueudet, G., \& Quéré, P.-V. (2018). "Making connections" in the mathematics courses for engineers: The example of online resources for trigonometry. In V. Durand-Guerrier, R. Hochmuth, S. Goodchild, \& N. M. Hogstad (Eds.), Proceedings of INDRUM 2018. Second conference of the international network for didactic research in university mathematics (pp. 135-144). Kristiansand, Norway: University of Agder and INDRUM.

Harris, D., Black, L., Hernandez-Martinez, P., Pepin, B., \& Williams, J. (2015). Mathematics and its value for engineering students: What are the implications for teaching? International Journal of Mathematical Education in Science and Technology, 46(3), 321-336.

Hochmuth, R., Biehler, R., \& Schreiber, S. (2014). Considering mathematical practices in engineering contexts focusing on signal analysis. In T. Fukawa-Connolly, G. Karakok, K. Keene, \& M. Zandieh (Eds.), Proceedings of the 17th annual conference on research in undergraduate mathematics education (pp. 693-698). Denver, CO: SIGMAA for Research in Undergraduate Mathematics Education.

Katz, V. J. (1998). A history of mathematics. An introduction (2nd ed.). Reading, MA: Addison Wesley Longman.

Kleiner, I. (1989). Evolution of the function concept: A brief survey. The College Mathematics Journal, 20(4), 282-300.

Kleiner, I. (2012). Excursions in the history of mathematics. New York: Springer.

Kreyzig, E. (2011). Advanced engineering mathematics (10th ed.). New York: Wiley.

Loch, B., \& Lamborn, J. (2016). How to make mathematics relevant to first-year engineering students: Perceptions of students on student-produced resources. International Journal of Mathematical Education in Science and Technology, 47(1), 29-44.

Lundheim, L. (2019). Fourierrekker [Fourier series]. Part of the module Spektrum til periodiske signal [Spectrum of periodic signals]. Trondheim, Norway: Department of Electronic Systems, NTNU Retrieved from https://www.youtube.com/watch?v=D0nf9AYOqoY.

Nilsson, J. W., \& Riedel, S. W. (2011). Electric circuits (9th ed.). Upper Saddle River, NJ: Pearson Education Inc.. O'Neil, P. V. (2018). Advanced engineering mathematics (8th ed.). Boston, MA: Cengage Learning.

Peters, J., Hochmuth, R., \& Schreiber, S. (2017). Applying an extended praxeological ATD-model for analyzing different mathematical discourses in higher engineering courses. In R. Göller, R. Biehler, R. Hochmuth, \& H.-G. Rück (Eds.), Didactics of mathematics in higher education as a scientific discipline. Conference proceedings (pp. 172-178). Kassel, Germany: University of Kassel.

Rudin, W. (1976). Principles of mathematical analysis (3rd ed.). Kogakusha, Japan: McGraw-Hill. 
Rüthing, D. (1984). Some definitions of the concept of function from Joh. Bernoulli to N. Bourbaki. The Mathematical Intelligencer, 6(4), 72-77.

Winkelman, P. (2009). Perceptions of mathematics in engineering. European Journal of Engineering Education, 34(4), 305-316.

Publisher's Note Springer Nature remains neutral with regard to jurisdictional claims in published maps and institutional affiliations. 